\title{
A 10-Gb/s Inductorless CMOS Limiting Amplifier With Third-Order Interleaving Active Feedback
}

\author{
Huei-Yan Huang, Jun-Chau Chien, Student Member, IEEE, and Liang-Hung Lu, Member, IEEE
}

\begin{abstract}
This paper presents an inductorless circuit technique for CMOS limiting amplifiers. By employing the third-order interleaving active feedback, the bandwidth of the proposed circuit can be effectively enhanced while maintaining a suppressed gain peaking within the frequency band. Using a standard $0.18-\mu \mathrm{m}$ CMOS process, the limiting amplifier is implemented for $10-\mathrm{Gb} / \mathrm{s}$ broadband applications. Consuming a DC power of $189 \mathrm{~mW}$ from a 1.8-V supply voltage, the fabricated circuit exhibits a voltage gain of $42 \mathrm{~dB}$ and a -3-dB bandwidth of $9 \mathrm{GHz}$. With a $2^{31}-1$ pseudo-random bit sequence at $10 \mathrm{~Gb} / \mathrm{s}$, the measured output swing and input sensitivity for a bit-error rate of $10^{-12}$ are 300 and $10 \mathrm{mV}_{\mathrm{pp}}$, respectively. Due to the absence of the spiral inductors, the chip size of the limiting amplifier including the pads is $0.68 \times 0.8 \mathrm{~mm}^{2}$ where the active circuit area only occupies $0.32 \times 0.6 \mathrm{~mm}^{2}$.
\end{abstract}

Index Terms-Active feedback, bandwidth enhancement techniques, broadband amplifiers, gain flatness, inductive peaking, limiting amplifiers, optical communications.

\section{INTRODUCTION}

$\mathbf{I}$ N THE RECEIVER front-end of an optical-fiber communication system, limiting amplifiers (LAs) operating at high data rates are mainly dominated by $\mathrm{SiGe}, \mathrm{GaAs}$, and $\mathrm{InP}$ technologies [1]-[3]. However, it is desirable to realize the LA circuits in standard CMOS processes which provide an unparalleled advantage in the implementation cost and system integration. Compared with the high-speed compound semiconductor devices, the inferior driving capability and the inherently low cut-off frequencies of the MOSFETs impose stringent limitations on the broadband amplifiers. Recently, circuit techniques have been proposed to enhance the bandwidth of CMOS LAs. By employing active inductors [4]-[6], Cherry-Hooper amplifiers [7], negative Miller capacitance and active feedback, LA circuits were successfully demonstrated at multi-gigahertz frequencies. However, for applications with a data rate beyond $10 \mathrm{~Gb} / \mathrm{s}$, inductive peaking technique is typically required to achieve the bandwidth specifications in the CMOS LA designs [8]-[11]. The excess circuit area occupied by the on-chip inductors is prohibitively large, especially when cascaded gain stages are employed, leading to a higher cost for the circuit implementation. In addition, the magnetic crosstalk among the peaking inductors and the $L C$-tank voltage-controlled oscillators may cause performance degradation of the clock and data recovery circuits in a fully integrated receiver front-end.

Manuscript received April 27, 2006; revised November 30, 2006.

The authors are with the Department of Electrical Engineering and Graduate Institute of Electronics Engineering, National Taiwan University, No. 1, Sec. 4 Roosevelt Road, Taipei, Taiwan 10617, R.O.C. (e-mail: lhlu@cc.ee.ntu.edu.tw).

Digital Object Identifier 10.1109/JSSC.2007.894819

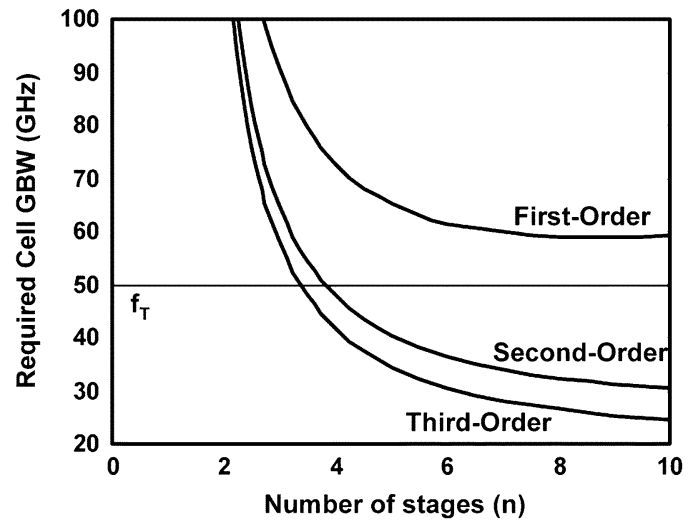

Fig. 1. The required gain-bandwidth product versus the number of stages for $A_{\mathrm{tot}}=40 \mathrm{~dB}$ and BW $\mathrm{BWt}_{\mathrm{tot}}=10 \mathrm{GHz}$.

In order to overcome the limitations resulted from the peaking inductors, an inductorless CMOS LA is presented. By incorporating the interleaving third-order active feedback in the cascaded gain stages, the LA bandwidth can be effectively enhanced while maintaining excellent gain flatness within the frequency band to suppress the overshoot at the output waveform. Due to the absence of on-chip inductors, a significant area reduction is thus achieved, making it extremely attractive for monolithic system integration. Using a $0.18-\mu \mathrm{m}$ CMOS process, the proposed circuit is implemented for the $10-\mathrm{Gb} / \mathrm{s}$ broadband applications.

Section II describes the design considerations of the active feedback in cascaded gain stages from a system aspect and analyzes the design trade-off of the third-order feedback loop. Section III presents the proposed interleaving feedback technique for bandwidth enhancement. The circuit design and the experimental results of the $10-\mathrm{Gb} / \mathrm{s}$ CMOS LA are shown in Sections IV and V, respectively. Finally, a conclusion is given in Section VI.

\section{LIMITING AMPLIFIER ARCHITECTURE}

\section{A. General Considerations of the Cascaded Gain Stages}

In conventional LA circuits, the architecture with cascaded gain stages is widely used to achieve a high gain and a broad bandwidth simultaneously. Assuming that the LA consists of $n$ identical cascaded gain stages with $m$ th-order Butterworth frequency response, a simplified analysis is performed to evaluate the gain and the bandwidth of the circuit. By extending the derivations in [10] and [12], the overall bandwidth of the cascaded stages is given by

$$
\mathrm{BW}_{\text {tot }}=\mathrm{BW}_{\text {cell }} \sqrt[2 m]{\sqrt[n]{2}-1}
$$



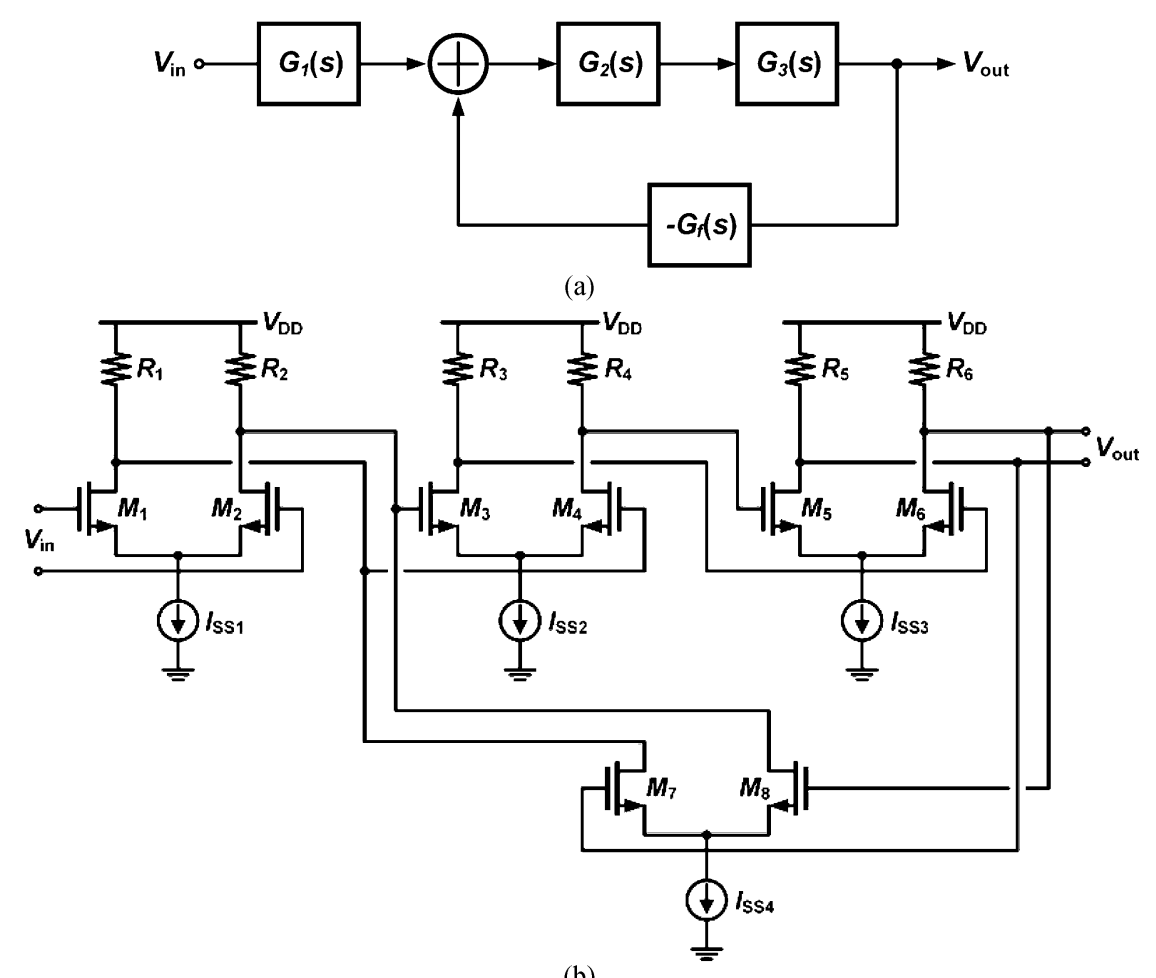

(b)

Fig. 2. (a) Block diagram and (b) circuit schematic of conventional third-order gain stages with active feedback.

where $\mathrm{BW}_{\text {cell }}$ is the bandwidth of the unit gain cells. Taking $\mathrm{BW}_{\text {tot }}=10 \mathrm{GHz}$ and $n=4$ as an example, the bandwidth $\mathrm{BW}_{\text {cell }}$ must exceed 23, 15, and $13 \mathrm{GHz}$ for $m=1,2$, and 3, respectively. Furthermore, the required gain-bandwidth product of the gain stage is

$$
\mathrm{GBW}_{\text {cell }}=\frac{\mathrm{BW}_{\text {tot }}}{\sqrt[2 m]{\sqrt[n]{2}-1}} \cdot \sqrt[n]{A_{\text {tot }}}
$$

where $A_{\text {tot }}$ represents the overall gain of the LA circuit.

Provided generic circuit specifications of the broadband LA design as $A_{\text {tot }}=40 \mathrm{~dB}$ and $\mathrm{BW}_{\text {tot }}=10 \mathrm{GHz}$, the required $\mathrm{GBW}_{\text {cell }}$ for the gain stages with various orders can be plotted as a function of $n$. For $m=1,2$, and 3, the results are shown in Fig. 1. It is observed that the required $\mathrm{GBW}_{\text {cell }}$ decreases as more identical stages are cascaded and tends to saturate when a large number of gain stages are utilized. The most straightforward approach to alleviate the limitations on the gain-bandwidth product of the cascaded stages is to use the largest $n$ possible. However, it results in a remarkable increase in power consumption, and the reduced gain per stage causes rapid accumulation of noise from the gain stages, leading to significant degradation in the input-referred noise figure of the amplifier. With practical design considerations, the maximum number of stages is typically limited within 5. In the following analysis, a stage number of 4 is chosen for the LA circuit to achieve a balanced performance between the gain-bandwidth product and the noise figure.

In addition to the number of stages, the required $\mathrm{GBW}_{\text {cell }}$ strongly depends on the order of the gain stages. According to the plots in Fig. 1, it is impossible to realize the LA specifications by cascading the first-order gain stages in a typical $0.18-\mu \mathrm{m}$ CMOS technology with $f_{T} \approx 50 \mathrm{GHz}$. As for the second and the third-order gain stages, the obtained gain-bandwidth products are 49 and $41 \mathrm{GHz}$, respectively, based on the four-stage LA architecture with $10-\mathrm{dB}$ voltage gain per stage. Although the required $\mathrm{GBW}_{\text {cell }}$ is greatly alleviated by adopting the second-order gain stages, it is still impractical to realize the circuit specifications with the $f_{T}$ provided. Typically, the inductive peaking technique is inevitable to further enhance the bandwidth of the second-order stages [10]. For a $10-\mathrm{Gb} / \mathrm{s}$ CMOS LA circuit without the peaking inductors, cascaded architecture with third-order gain stages are investigated with theoretical derivations.

\section{B. Analysis of Third-Order Gain Stages With Active Feedback}

A generic block diagram of the third-order gain stage with active feedback is illustrated in Fig. 2(a) where $G_{1}, G_{2}$, and $G_{3}$ are the gain cells and $G_{f}$ is the feedback cell. By using $G_{f}$ to return a fraction of the output signal to the input of $G_{2}$, the third-order system can be characterized by the transfer function

$$
H_{3 \mathrm{rd}}(s)=\frac{G_{1}(s) \cdot G_{2}(s) \cdot G_{3}(s)}{1+G_{2}(s) \cdot G_{3}(s) \cdot G_{f}(s)} .
$$

For circuit implementations, the gain and the feedback cells are conventionally realized by CMOS differential pairs with resistive loads as shown in Fig. 2(b). To simplify the analysis, the gain and feedback cells are approximated by the single-pole stages as

$$
G_{1}(s)=G_{2}(s)=G_{3}(s)=G(s)=\frac{G_{m} R}{1+s R C}
$$




$$
G_{f}(s)=\frac{G_{m f} R}{1+s R C}
$$

where $R$ and $C$ are the resistive and capacitive loads of the differential pairs, respectively, and the transconductances of the cells are represented by $G_{m}$ and $G_{m f}$. By substituting (4) and (5) into (3), the overall transfer function of the third-order gain stage is given by

$$
\begin{aligned}
H_{3 \mathrm{rd}}(s) & =\frac{G_{m}^{3} R^{3}}{(1+s R C)^{3}+G_{m}^{2} G_{m f} R^{3}} \\
& =\frac{A_{0}^{3}}{\left(1+s / \omega_{0}\right)^{3}+A_{0}^{2} \beta}
\end{aligned}
$$

where $\omega_{0}=1 / R C, A_{0}=G_{m} R$ and $\beta=G_{m f} R$. The resulting low-frequency gain of the third-order system is

$$
A_{v 0}=\frac{A_{0}^{3}}{1+A_{0}^{2} \beta} .
$$

Typically, such third-order systems can be characterized by one non-dominant real pole and two dominant complex conjugate poles as

$$
\begin{aligned}
\omega_{p 1} & =-\omega_{0}\left(1+\left(A_{0}^{2} \beta\right)^{\frac{1}{3}}\right) \\
\omega_{p 2,3} & =-\omega_{0}\left(1-\frac{1}{2}\left(A_{0}^{2} \beta\right)^{\frac{1}{3}} \pm j \frac{\sqrt{3}}{2}\left(A_{0}^{2} \beta\right)^{\frac{1}{3}}\right) .
\end{aligned}
$$

Since the maximum flat behavior is not applicable in the analysis of the third-order gain stages, a numerical approach is employed to evaluate the frequency response by solving the following equation:

$$
\begin{aligned}
& \left|H_{3 \mathrm{rd}}\left(j \omega_{3 d B}\right)\right| \\
& =\frac{A_{0}^{3}}{\sqrt{\left[1+A_{0}^{2} \beta-3\left(\frac{\omega_{3 d B}}{\omega_{0}}\right)^{2}\right]^{2}+\left[3\left(\frac{\omega_{3 d B}}{\omega_{0}}\right)-\left(\frac{\omega_{3 d B}}{\omega_{0}}\right)^{3}\right]^{2}}} \\
& =\frac{A_{v 0}}{\sqrt{2}} .
\end{aligned}
$$

The normalized $-3-\mathrm{dB}$ bandwidth and the low-frequency gain are shown in Fig. 3(a) as a function of the feedback gain $\beta$. It is noted that the $-3-\mathrm{dB}$ bandwidth increases with the feedback gain at the cost of a reduced $A_{v 0}$. Moreover, the associated peaking frequency $\left(\omega_{\text {peak }}\right)$ and the amount of gain peaking can be derived by calculating

$$
\frac{d}{d \omega}\left|H_{3 r d}(j \omega)\right|_{\omega=\omega_{\text {peak }}}=0
$$

and

$$
\operatorname{Peaking}(\mathrm{dB})=20 \log \left(\frac{\left|H_{3 \mathrm{rd}}\left(j \omega_{\text {peak }}\right)\right|}{A_{v 0}}\right)
$$

respectively. Fig. 3(b) shows the amount of gain peaking as a function of $\beta$, indicating that the amount of gain peaking also increases with the feedback gain. With $\beta=0.3$, the bandwidth

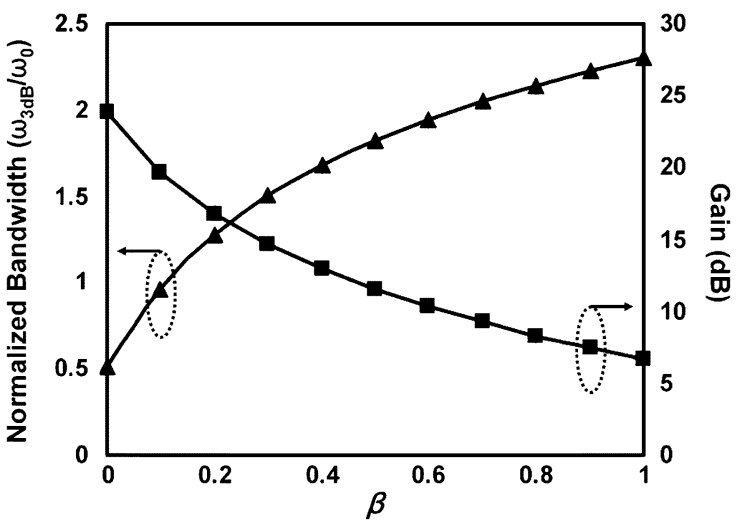

(a)

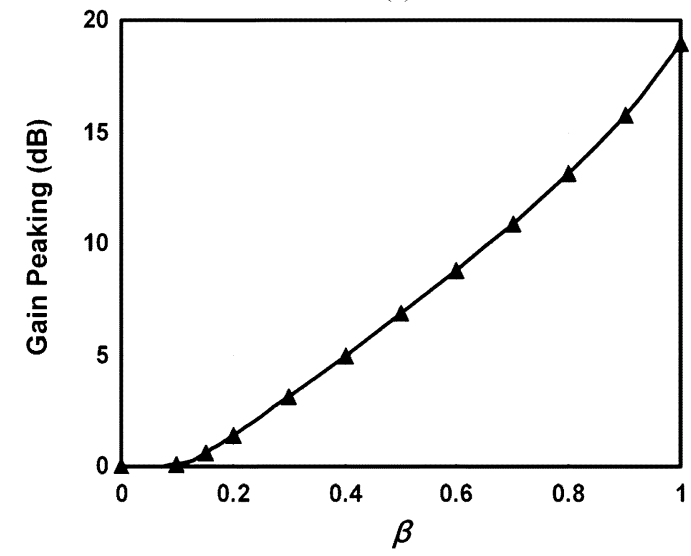

(b)

Fig. 3. (a) Simulated gain-bandwidth characteristics and (b) the associated gain peaking of conventional 3rd-order gain stages with active feedback. $\left(A_{0}=2.5\right)$.

of the third-order gain stage improves by a factor of 1.5 accompanied by a gain peaking of $3.14 \mathrm{~dB}$ in the frequency response. However, as more gain stages are cascaded, the gain peaking in the overall LA frequency response accumulates rapidly, making it virtually impossible to meet the requirement in the OC-192 specifications. Considering the associated gain peaking, it is evident that using the third-order gain stages with the conventional feedback topology for the LA designs is unrealistic even though the bandwidth is effectively enhanced. Therefore, in order to take the advantage of the high-order gain stages, novel feedback techniques have to be developed to suppress the gain peaking while maintaining the broadband characteristics.

\section{Proposed Interleaving FeEdBack Topology}

To overcome the severe gain peaking resulted from the thirdorder gain stages, an interleaving feedback technique is proposed. Assuming that identical gain cells and feedback cells as specified in (4) and (5) are used, the block diagram of the conventional cascaded architecture is shown in Fig. 4(a) while the proposed circuit topology with additional feedback between the two adjacent stages is illustrated in Fig. 4(b). In this section, the influence of the interleaving feedback on the system behavior will be presented.

\section{A. Bandwidth Enhancement and Gain Peaking Reduction}

The analysis starts with the examination of the system behavior for conventional cascaded third-order gain stages. From 


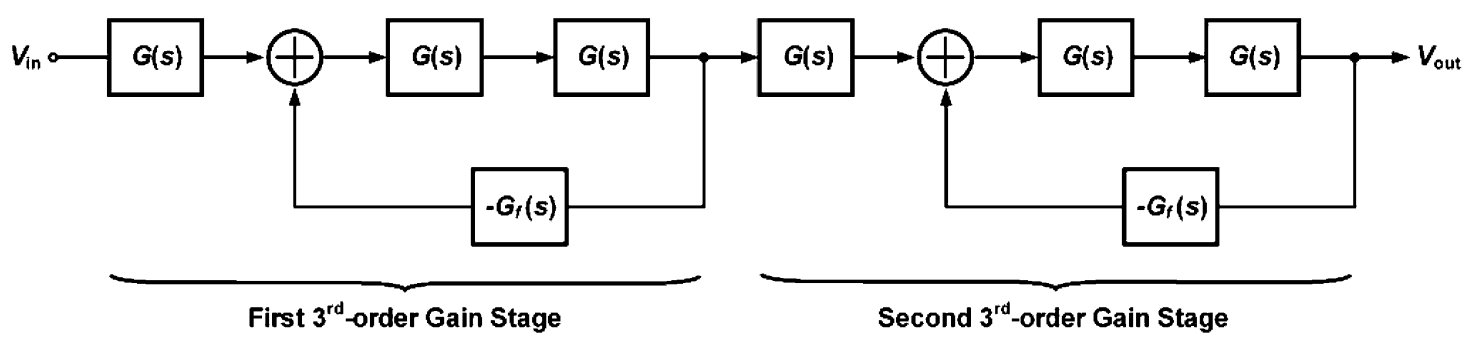

(a)

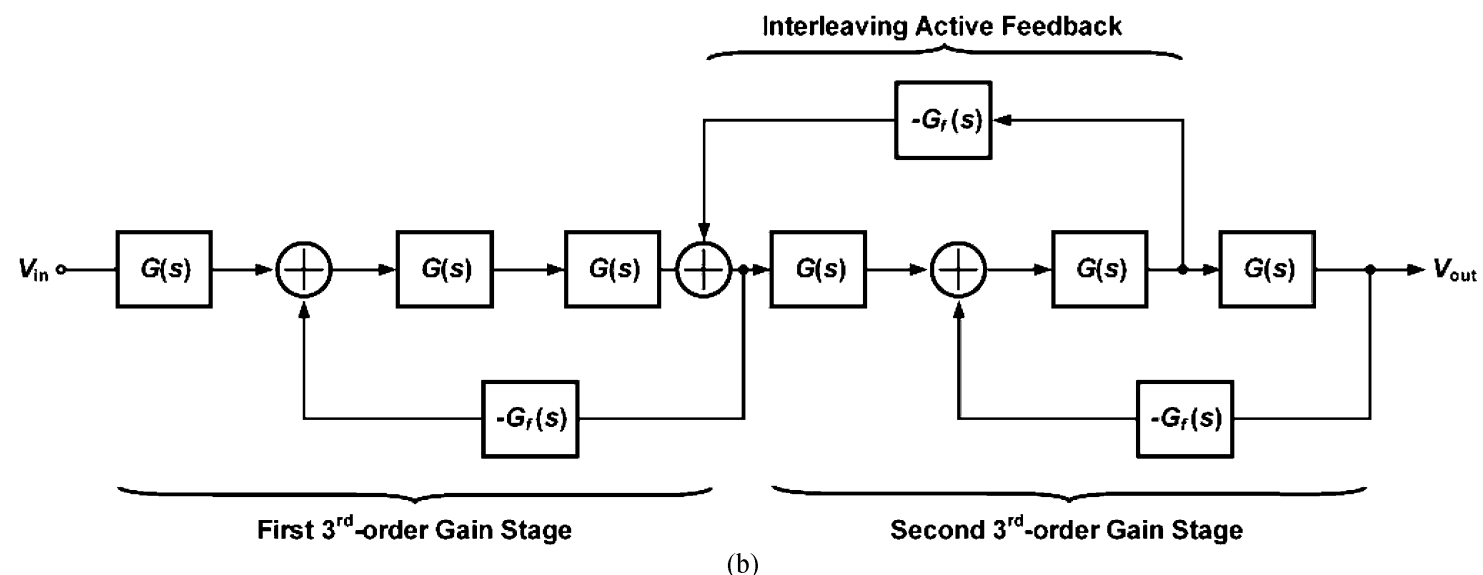

(b)

Fig. 4. Block diagrams of (a) the conventional cascaded third-order stages and (b) the cascaded third-order stages with the proposed interleaving feedback.

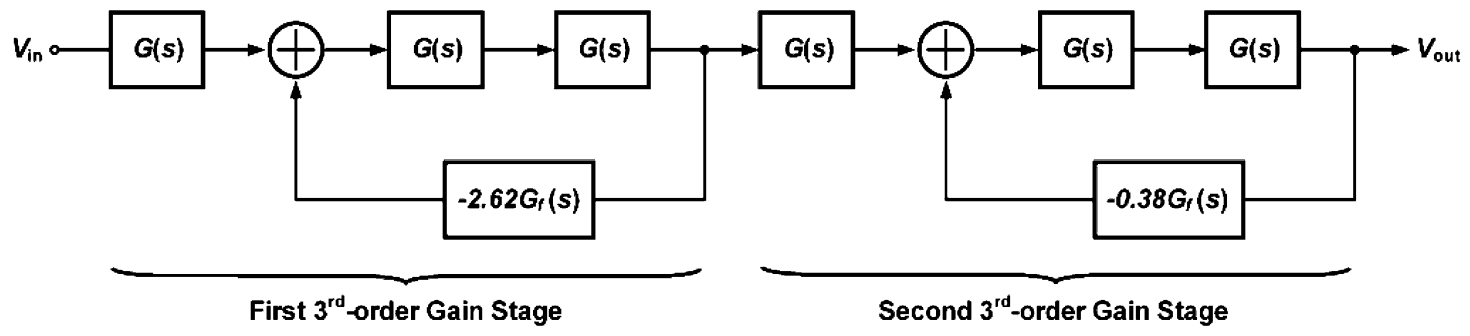

Fig. 5. Equivalent system block diagram of the proposed topology.

the block diagram as shown in Fig. 4(a), the transfer function can be expressed as

$$
\begin{aligned}
H_{1}(s) & =\frac{G^{3}(s)}{1+G^{2}(s) G_{f}(s)} \cdot \frac{G^{3}(s)}{1+G^{2}(s) G_{f}(s)} \\
& =\frac{G^{6}(s)}{1+2 G^{2}(s) G_{f}(s)+G^{4}(s) G_{f}^{2}(s)} .
\end{aligned}
$$

On the other hand, the transfer function of the cascaded stages with the proposed interleaving feedback topology, as shown in Fig. 4(b), is given by

$$
\begin{aligned}
H_{2}(s) & =\frac{G^{6}(s)}{1+3 G^{2}(s) G_{f}(s)+G^{4}(s) G_{f}^{2}(s)} \\
& =H_{A}(s) \cdot H_{B}(s)
\end{aligned}
$$

where

$$
H_{A}(s)=\frac{G^{3}(s)}{1+2.62 G^{2}(s) G_{f}(s)}
$$

$$
H_{B}(s)=\frac{G^{3}(s)}{1+0.38 G^{2}(s) G_{f}(s)}
$$

It is noted that the transfer function of the proposed topology can be equivalently decomposed into two conventional third-order stages with different gains at the feedback networks as shown in Fig. 5. As the feedback gain varies, the pole splitting effect causes the three poles of the third-order stages deviate from the locations as specified in (8) and (9). Fig. 6 illustrates the pole splitting behavior of the interleaving feedback topology with respective to $\beta$. Based on the transfer function in (14), the simulated frequency response with $\beta=0.3$ is plotted in Fig. 7. Because of the non-uniform architecture of the cascaded stages, the overall gain flatness maintains within $\pm 0.5 \mathrm{~dB}$ even though the frequency response of the individual stages exhibits a gain peaking up to $3 \mathrm{~dB}$. The simulated frequency responses of the cascaded third-order stages are shown in Fig. 8. It is observed that, due to the use of the interleaving active feedback, the bandwidth of the cascaded third-order gain stages is improved by $18 \%$ at the expense of $2-\mathrm{dB}$ degradation in the voltage gain. Furthermore, the excess gain peaking near the roll-off frequency is effectively suppressed, exhibiting excellent gain flatness within the entire frequency band. Note that the concept of 


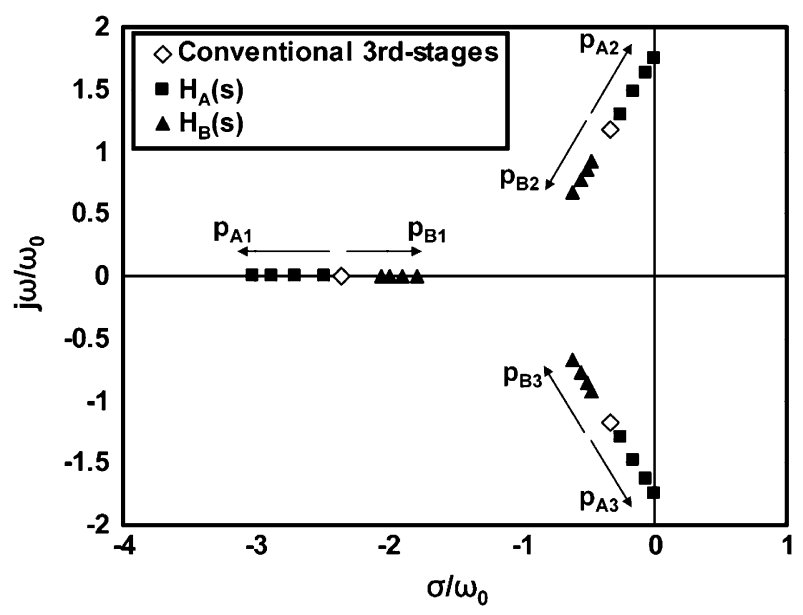

Fig. 6. Pole splitting behavior of the proposed feedback technique.

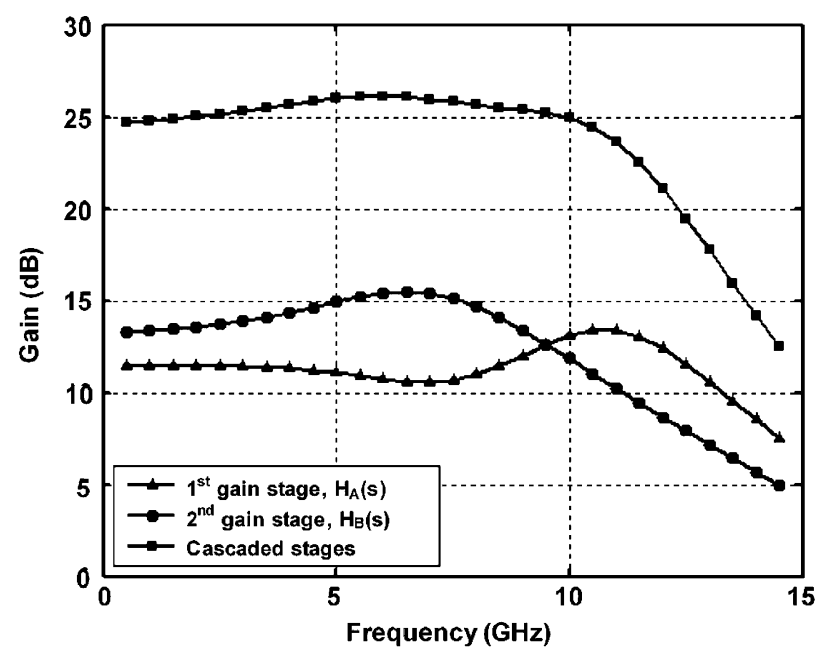

Fig. 7. Simulated frequency response of the cascaded architecture with nonuniform third-order gain stages.

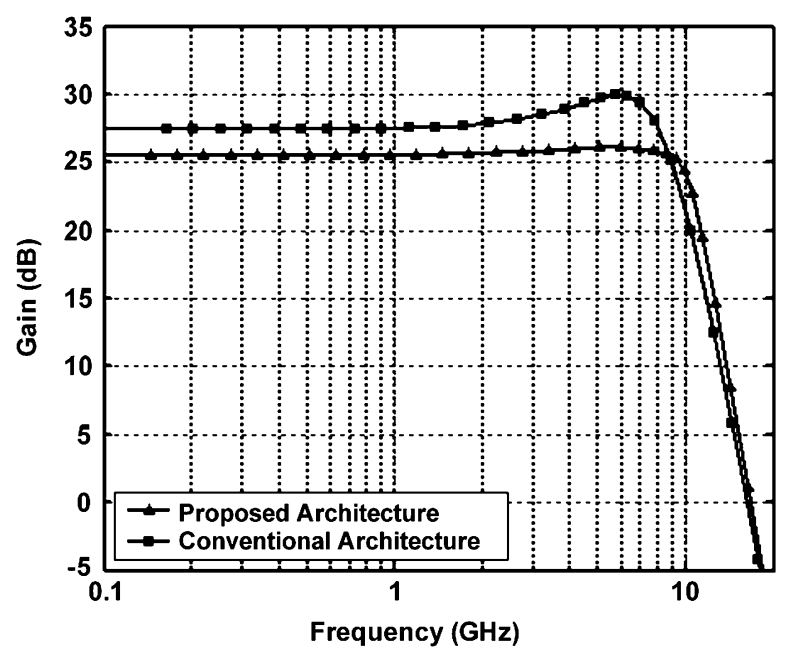

Fig. 8. Simulated frequency response of the cascaded third-order gain stages.

stagger tuning of cascaded multi-stage amplifier described here is similar to the design as demonstrated in [13].
In addition to the analysis on gain peaking, the performance in terms of the overshoot and ripple is studied as well. With a 10-Gb/s pseudo-random bit sequence (PRBS), the simulated output eye diagrams for various cascaded architectures are demonstrated in Fig. 9. It is evident that a clear eye diagram is not available for the cascaded first-order gain stages since the overall bandwidth is far below the specification. By using the second-order gain stages, the bandwidth is effectively enhanced, but it is still insufficient to operate at a $10-\mathrm{Gb} / \mathrm{s}$ data rate without peaking inductors. As for the results from conventional third-order gain stages, the eye opening is significantly improved due to the extended bandwidth. However, the excess gain peaking leads to unacceptable overshoot and ripple. For the cascaded third-order gain stages with the interleaving feedback technique, the simulated eye diagram indicates optimized output characteristics compared with the other cascaded architectures.

\section{B. Practical Design Considerations}

Although the non-uniform cascaded topology, as shown in Fig. 5, demonstrates identical system behavior as the proposed topology with the interleaving active feedback, it is not preferable for the circuit implementation. In order to avoid gain peaking accumulation, the ratio of the feedback gains between the two distinct third-order stages has to be sufficiently large for the design of broadband amplifiers. Limited by the specification of the overall gain, the transconductance in the feedback cell of the gain stage with lower roll-off frequency becomes extremely small, making it impractical for circuit realization and very sensitive to process variation. Therefore, the proposed circuit topology which is composed of uniform gain and feedback cells is well suited for the 10-Gb/s LA design.

Based on the pole splitting behavior as depicted in Fig. 6, a pair of conjugate poles may enter the right half-plane with excessively large loop gain, causing system instability. In order to investigate the circuit stability of the proposed interleaving feedback architecture, the real part of the complex conjugate poles from (15) is expressed as

$$
\operatorname{Re}\left\{p_{A 2}\right\}=\operatorname{Re}\left\{p_{A 3}\right\} \approx-\omega_{0}\left(1-0.69\left(A_{0}^{2} \beta\right)^{\frac{1}{3}}\right) .
$$

From (17), the loop gain $\left(A_{0}^{2} \beta\right)$ has to be smaller than 3 to keep the poles from moving into the right half-plane. For circuit implementations, a design margin is required in choosing $G_{m}$ and $G_{m f}$ of the cascaded stages to ensure the circuit stability against process and temperature variations.

\section{Circuit Design}

By employing the proposed circuit technique, an inductorless LA is implemented in a $0.18-\mu \mathrm{m}$ CMOS process for $10-\mathrm{Gb} / \mathrm{s}$ applications. Fig. 10 illustrates the architecture of the fully integrated LA which is composed of an amplifier core, an offset cancellation feedback and an output buffer.

\section{A. Amplifier Core}

To achieve the required gain and bandwidth specifications in the inductorless LA design, four cascaded third-order gain 


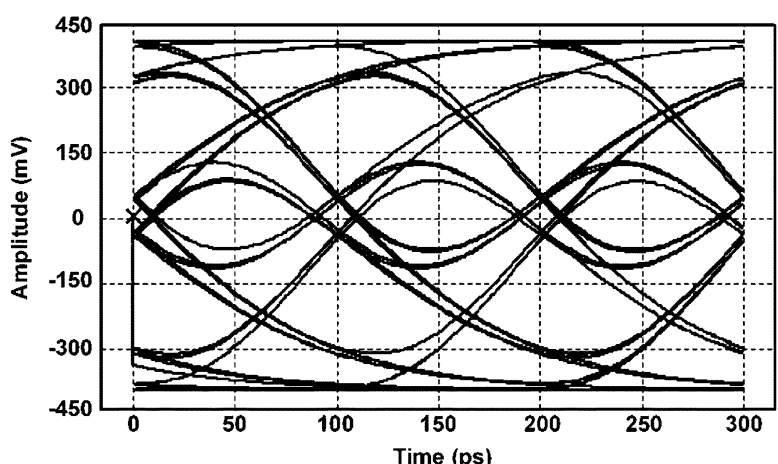

(a)

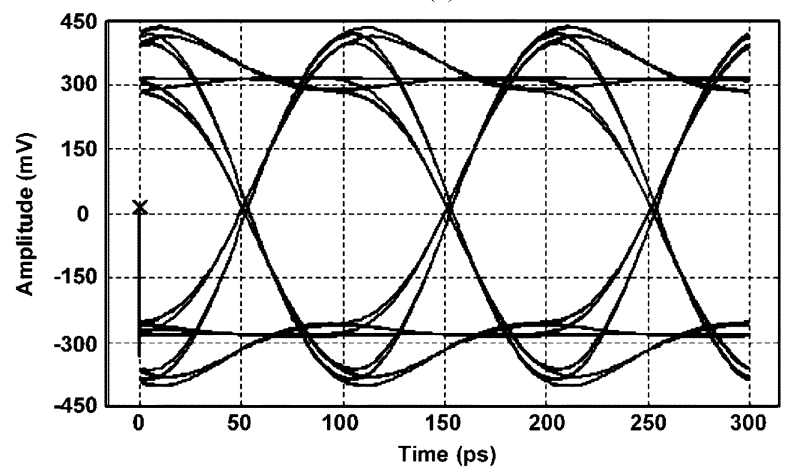

(c)

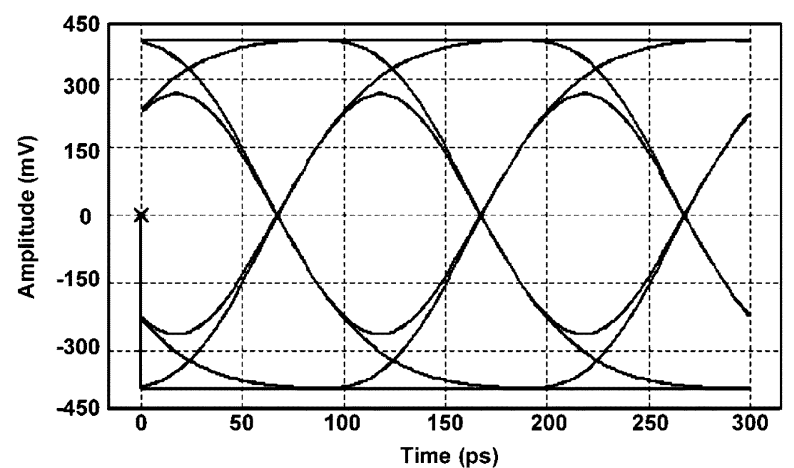

(b)

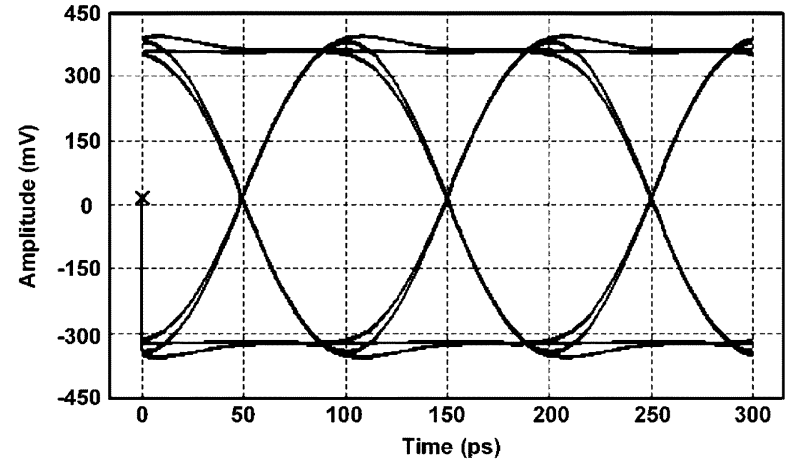

(d)

Fig. 9. Simulated eye diagrams of the cascaded amplifier operating at a 10-Gb/s PRBS for (a) first-order gain stages, (b) second-order gain stages, (c) third-order gain stages, and (d) third-order gain stages with the interleaving feedback technique.

Fig. 10. Architecture of the 10-Gb/s CMOS limiting amplifier.

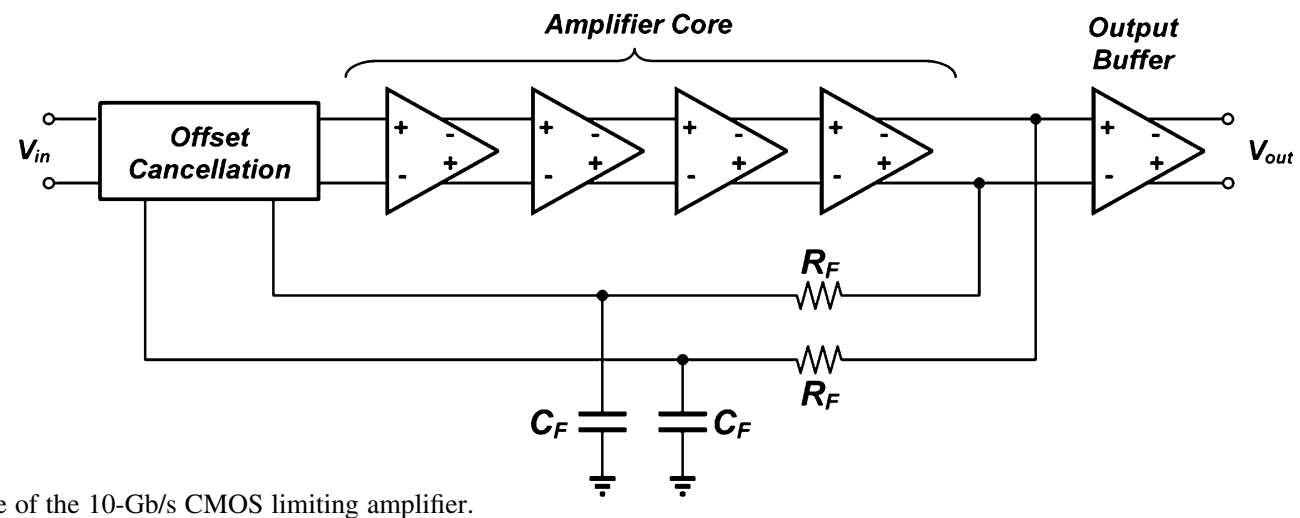

stages with interleaving active feedback are utilized for the amplifier core as shown in Fig. 11. The gain cells are realized by identical differential pairs with resistive load $R$ while the active feedback is provided by the $G_{m f}$ cells. Considering the specifications such as time constant, voltage gain and output swing, a $200-\Omega$ resistive load and $G_{m}$ of $12.5 \mathrm{mS}$ are employed for the differential pairs. Based on the prediction in Fig. 3, the designated $G_{m f}$ of the third-order gain stage in this particular design is set to $1.5 \mathrm{mS}$, leading to a feedback gain of 0.3 for the required bandwidth enhancement with moderate gain peaking. Note that the transistor sizes for the $G_{m}$ cells are substantially larger than those for the $G_{m f}$ cells. Therefore, the loading effect due to the interleaving feedback cells can be neglected, and the derivations in Section III are still valid for the circuit design. After optimization of the third-order gain stage, the amplifier core is realized by cascading four of these blocks with active feedback cells be- tween every two adjacent stages. The design values are listed in Table I.

With the design parameters of the $G_{m}$ and $G_{m f}$ cells, a transistor-level Monte Carlo simulation was performed on the proposed amplifier architecture to evaluate the circuit stability. Provided a $\pm 20 \%$ variation in the load-resistance, a $\pm 10 \%$ variation in transistor aspect ratios and a $\pm 10 \%$ variation in supply voltage for a temperature range from 0 to $100^{\circ}$, the simulated results are shown in Fig. 12. It is observed that the maximum loop gain is bounded within 2.2 for a nominal value of 1.9 , exhibiting sufficient safe margin to ensure the system stability of the amplifier core.

\section{B. Offset Cancellation}

In the LA architecture, an offset cancellation network with high-pass characteristics is employed to prevent the amplifier 

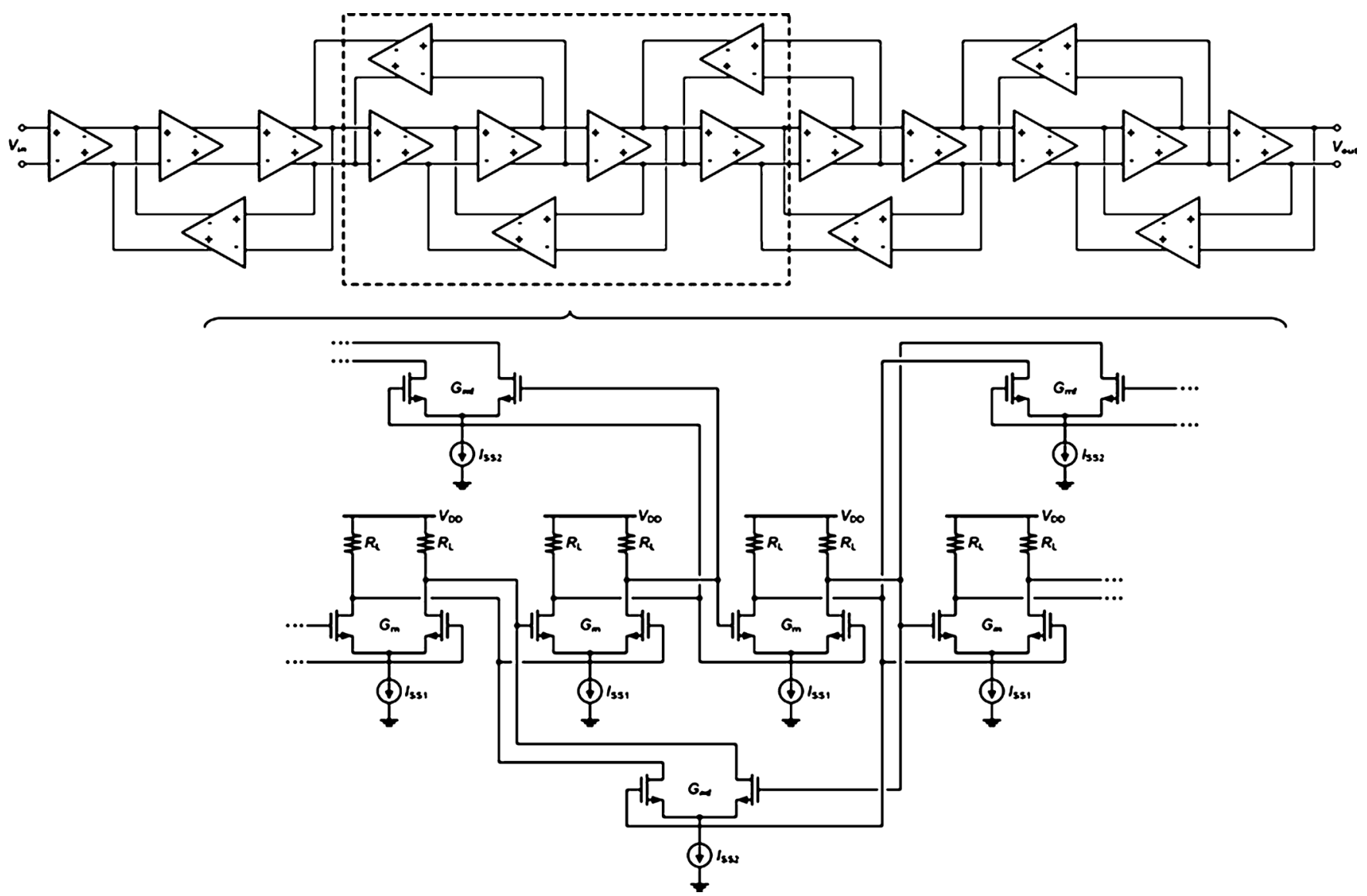

Fig. 11. Circuit schematic of the amplifier core with the cascaded third-order gain stages and the interleaving active feedback.

TABLE I

DESIGN PARAMETERS OF THE AMPLIFIER CORE

\begin{tabular}{|c|c|}
\hline \multicolumn{2}{|c|}{ Design Parameters } \\
\hline$(W / L)_{\mathrm{Gm}}$ & $30 \mu \mathrm{m} / 0.18 \mu \mathrm{m}$ \\
$(W / L)_{\mathrm{Gmf}}$ & $5 \mu \mathrm{m} / 0.18 \mu \mathrm{m}$ \\
$I_{s s 1}$ & $6.0 \mathrm{~mA}$ \\
$I_{s s 2}$ & $0.5 \mathrm{~mA}$ \\
$R_{\mathrm{L}}$ & $200 \Omega$ \\
\hline
\end{tabular}

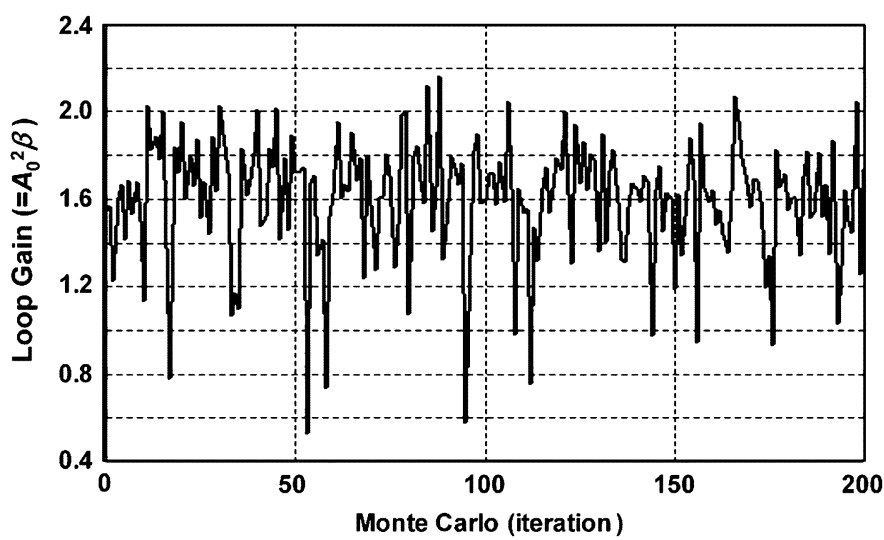

Fig. 12. Variation in the loop-gain of the third-order gain stages from the transistor-level Monte Carlo simulations. from being saturated by the offset voltage due to device mismatch [10]. By incorporating a feedback gain stage and a passive low-pass filter with the amplifier core as shown in Fig. 13, the corner frequency of the resulting high-pass response is

$$
f_{c}=\frac{A_{C} A_{F}+1}{2 \pi \cdot C_{F} R_{F}}
$$

where $A_{C}$ and $A_{F}$ are the gains of the amplifier core and the offset cancellation feedback, respectively. $R_{F}$ and $C_{F}$ are the passive elements used in the low-pass filter to extract the output DC level of the amplifier core. Note that the corner frequency $f_{c}$ must fall in the range of a few tens of kilohertz to eliminate the output droop in the presence of long runs. As a result, the required resistance and capacitance values are enormous in typical LA designs. To minimize the chip area occupied by the passive elements, pMOS transistors operating in the triode region are employed as $R_{F}$, and $C_{F}$ is realized by nMOS transistors. The transconductance cell in the feedback path is incorporated with the first gain cell of the amplifier core, acting as a DC subtractor circuit for offset cancellation.

\section{Output Buffer}

To drive the testing instruments with an input impedance of $50 \Omega$, an output buffer is included in the design. Considering the inherently low equivalent impedance at the output node, the buffer has to steer a current as high as $20 \mathrm{~mA}$ to maintain a sufficient output swing. However, the use of large transistor sizes results in excess capacitive loading to the amplifier core, making 


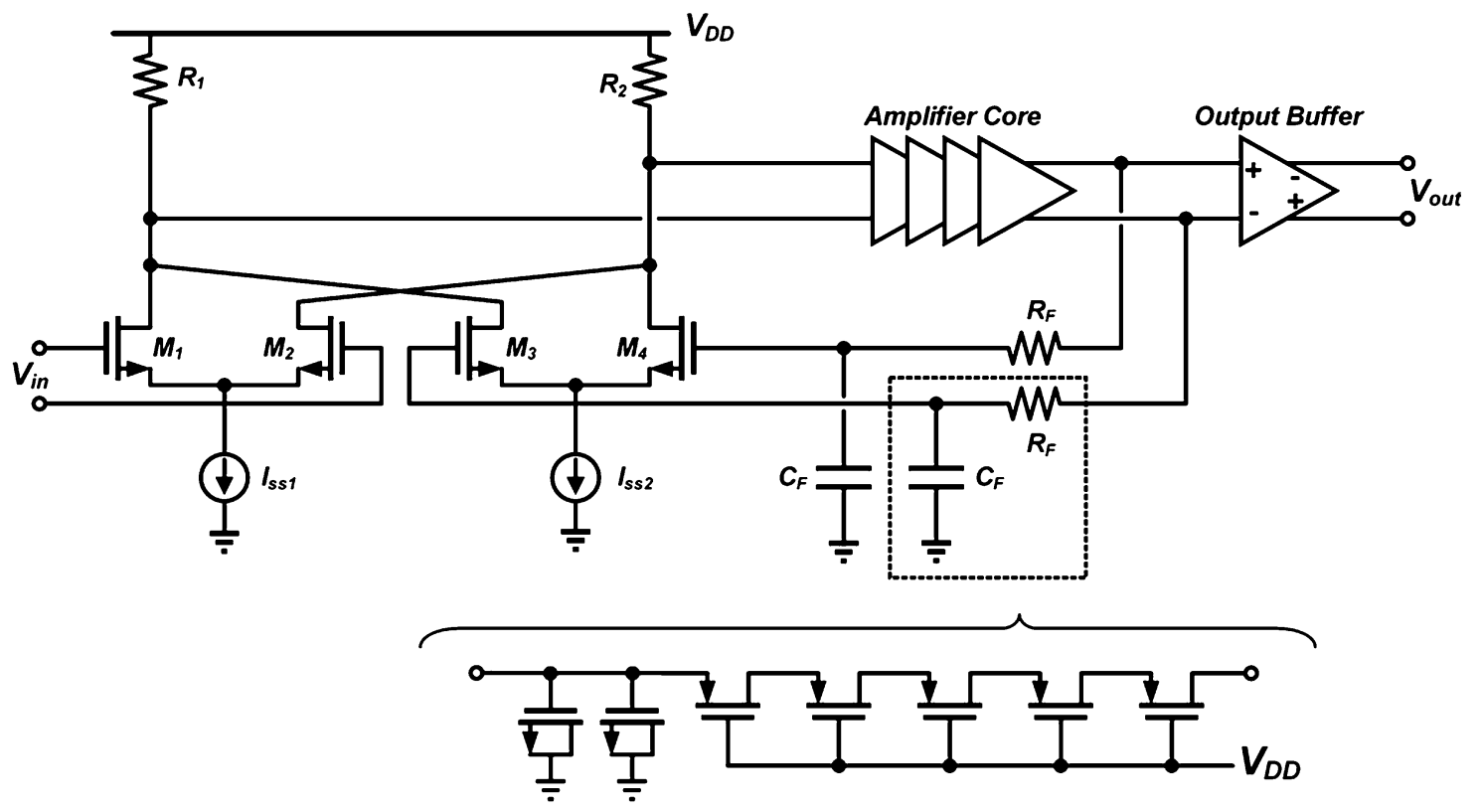

Fig. 13. Feedback network for offset cancellation.

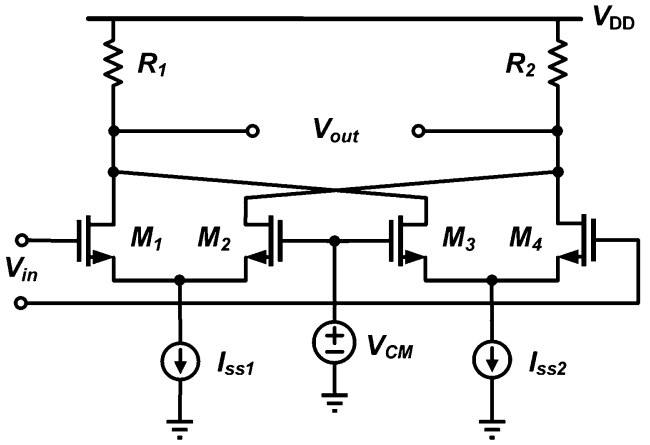

Fig. 14. Circuit schematic of the output buffer.

it extremely difficult to satisfy the overall bandwidth requirement. In order to overcome the bandwidth limitation, a $f_{T}$ doubler circuit [10], which reduces the capacitive loading by half, is employed as the output buffer as shown in Fig. 14.

\section{EXPERIMENTAL RESULTS AND DISCUSSION}

The microphotograph of the fabricated LA is shown in Fig. 15. Due to the absence of peaking inductors for the gain stages, the total chip size with the pads measures $0.68 \times 0.8 \mathrm{~mm}^{2}$ where active circuit only occupies an area of $0.32 \times 0.6 \mathrm{~mm}^{2}$. The circuit performance was characterized by on-wafer probing.

Operated at a supply voltage of $1.8 \mathrm{~V}$, the LA with the on-chip bias circuitry draws a DC current of $105 \mathrm{~mA}$. The small-signal characteristics of the circuit are evaluated by the S-parameter measurement as shown in Fig. 16. The LA exhibits a differential voltage gain of $42 \mathrm{~dB}$ with a $-3-\mathrm{dB}$ frequency of $9 \mathrm{GHz}$ while maintaining input and output return losses better than $10 \mathrm{~dB}$. Due to the use of the interleaving active feedback, the measured gain ripple is $\pm 0.8 \mathrm{~dB}$ within the entire frequency band.

The output eye diagram and the bit-error rate (BER) of the LA were characterized by an Agilent N4901A BERT system and an

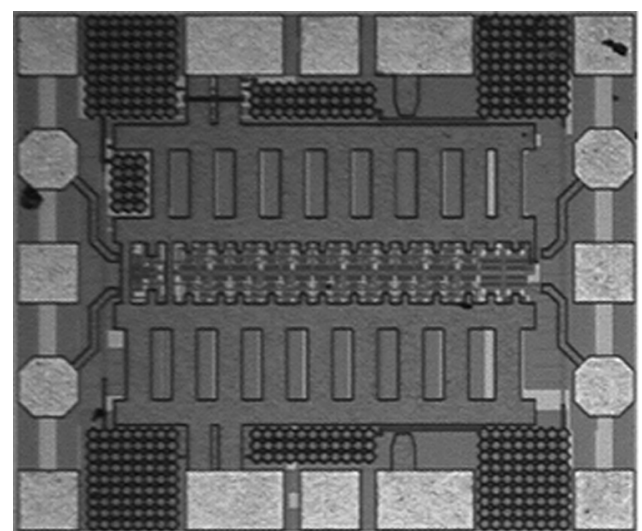

Fig. 15. Microphotograph of the fabricated limiting amplifier.

Agilent $86100 \mathrm{C}$ oscilloscope. With a $2^{31}-1$ PRBS at $10 \mathrm{~Gb} / \mathrm{s}$, the output eye diagrams with various input voltage swings are shown in Fig. 17, indicating an output swing of $300 \mathrm{mV}$ and a vertical eye opening greater than $200 \mathrm{mV}$. The measured BER versus input voltage swing is shown in Fig. 18, where the fabricated LA demonstrates an input sensitivity of $10 \mathrm{mV}_{\mathrm{pp}}$ for $\mathrm{BER}=10^{-12}$. The performance of the proposed LA circuit and the comparison with prior works are summarized in Table II.

The analysis of the third-order active feedback system presented in Section II is based on the assumption that the gain cells are in linear operation with transfer functions as shown in (4) and (5). In reality, the circuit characteristics are strongly influenced by the signal amplitude. As the signal propagates through cascaded stages, the gain cells are eventually saturated by the increasing voltage swing, and act as unity-gain buffers instead. As a result, the feedback loop gain falls below unity due to $G_{m f}$ and the bandwidth are dominated by the time constant. Simulation results reflecting the saturation of the gain cells are depicted in Fig. 19. By employing the inverse scaling technique for the cascaded gain cells [4], the circuit performance of the LA can be further improved. However, it complicates the system analysis 


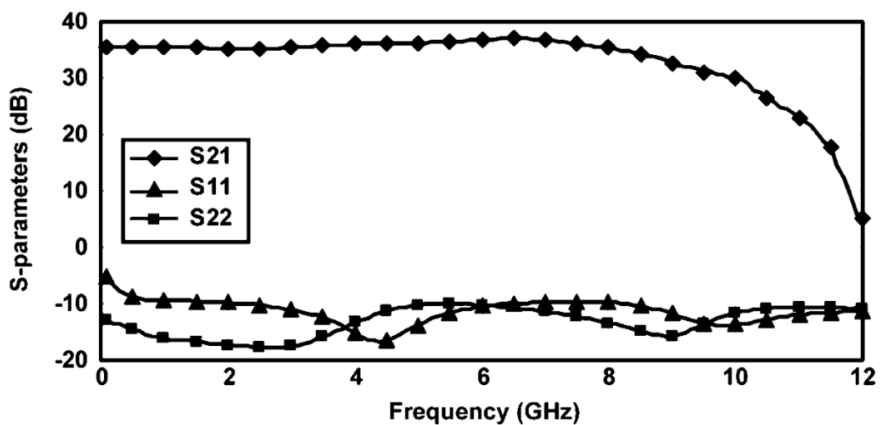

(a)

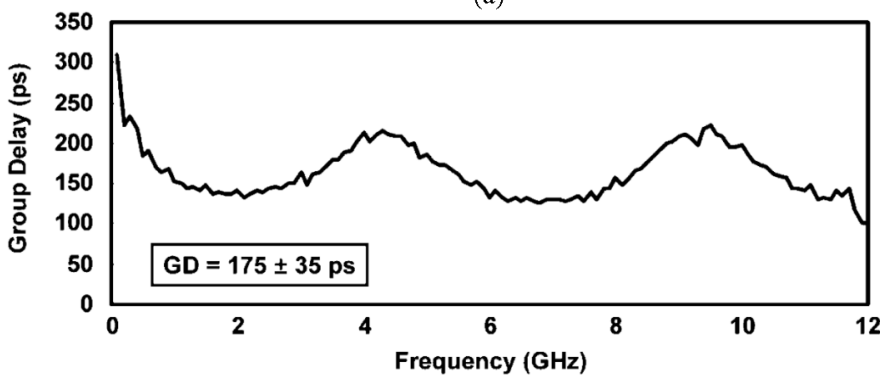

(b)

Fig. 16. Measured (a) S-parameters and (b) group delay of the fabricated limiting amplifier.

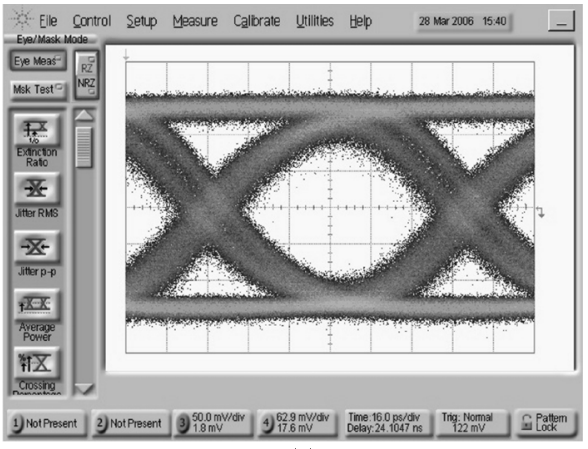

(a)

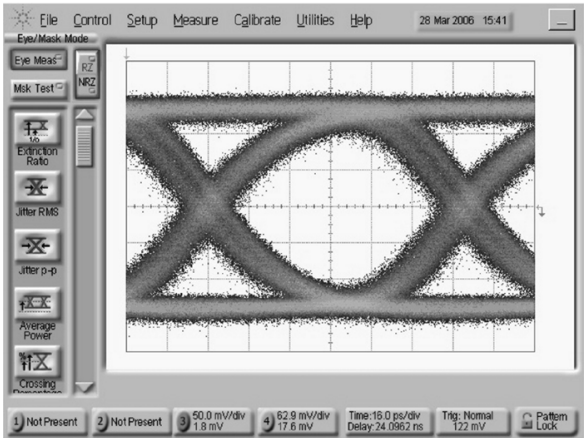

(b)

Fig. 17. The measured output eye diagrams for an input level of (a) $10 \mathrm{mV}_{\mathrm{pp}}$ and (b) $100 \mathrm{mV}_{\mathrm{pp}}$. (Horizontal scale: $16 \mathrm{ps} /$ div., vertical scale: $50 \mathrm{mV} / \mathrm{div}$.).

and circuit design, especially when the interleaving feedback technique is applied.

\section{CONCLUSION}

In this paper, a novel circuit technique is presented for CMOS LA designs. With the interleaving third-order active feedback, the proposed circuit demonstrates an enhanced $-3-\mathrm{dB}$ bandwidth with excellent gain flatness over the frequency band.

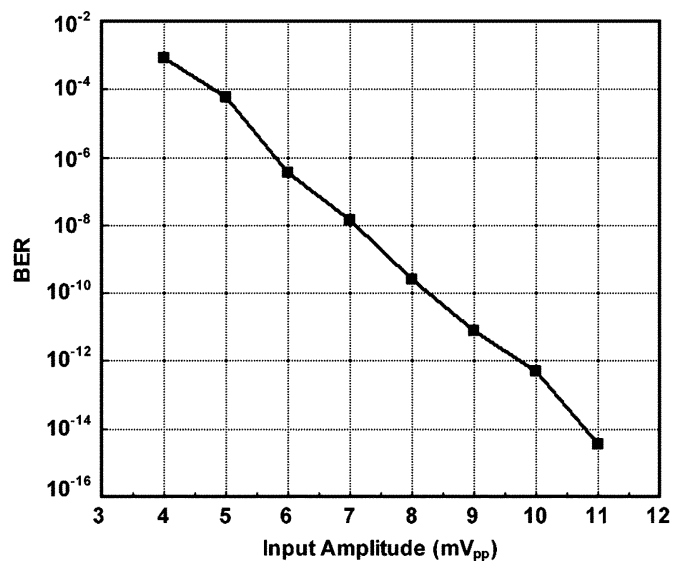

Fig. 18. Measured BER versus input signal level with $2^{31}-1$ PRBS.

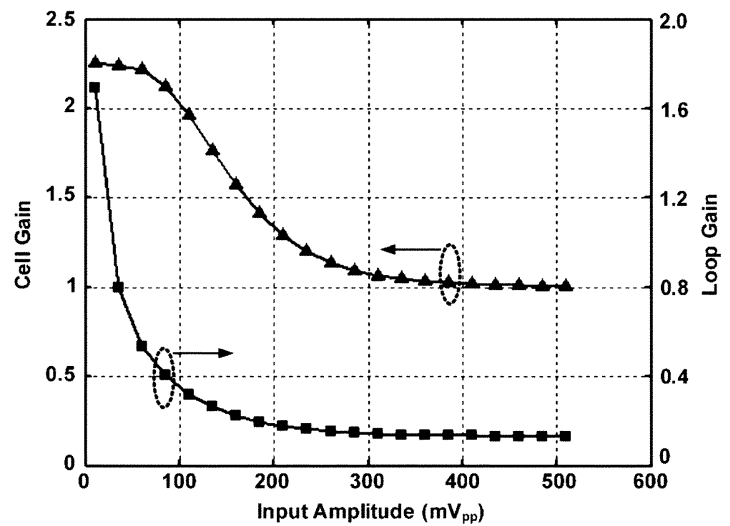

(a)

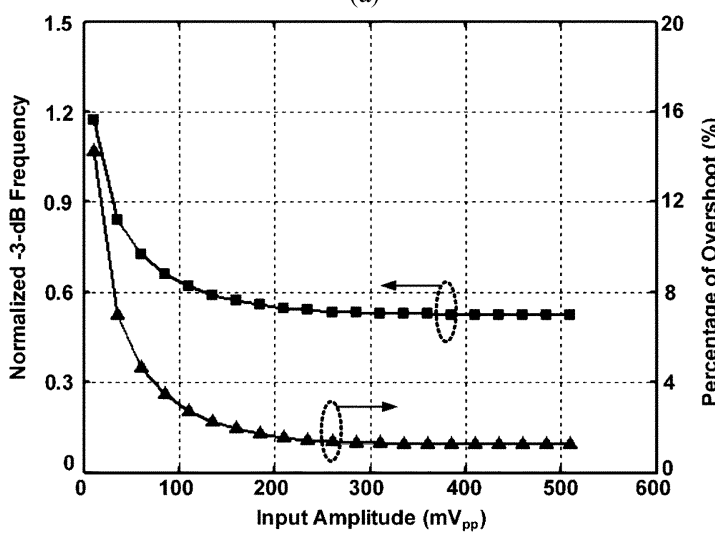

(b)

Fig. 19. Simulated performance of the gain cells versus input signal level.

Using a standard $0.18-\mu \mathrm{m}$ CMOS process, an inductorless $10-\mathrm{Gb} / \mathrm{s} \mathrm{LA}$ is designed and fabricated, exhibiting a voltage gain of $42 \mathrm{~dB}$ and a bandwidth of $9 \mathrm{GHz}$. Due to the absence of inductors in the circuit implementation, the active area of the LA measures only $0.32 \times 0.6 \mathrm{~mm}^{2}$. It is extremely suitable for monolithic system integration with the receiver front-end for low-cost optical-fiber applications.

\section{ACKNOWLEDGMENT}

The authors would like to thank National Chip Implementation Center and National Nano Device Laboratories for chip fabrication and technical support. The authors are also grateful to Chih-Fan Liao for help with the chip measurement. 
TABLE II

PERFORMANCE COMPARISON OF THE LIMITING AMPLIFIERS

\begin{tabular}{|c|c|c|c|c|c|c|}
\hline Ref. & [1] & [4] & [8] & [10] & [11] & This \\
\hline Tech. & $\begin{array}{c}\text { SiGe HBT } \\
f_{T}=47 G H z\end{array}$ & $\begin{array}{c}0.25-\mu \mathrm{m} \\
\text { cMOS }\end{array}$ & $\begin{array}{c}0.18-\mu \mathrm{m} \\
\text { CMOS }\end{array}$ & $\begin{array}{c}0.18-\mu m \\
\text { CMOS }\end{array}$ & $\begin{array}{c}0.13-\mu \mathrm{m} \\
\text { CMOS }\end{array}$ & $\begin{array}{c}0.18-\mu \mathrm{m} \\
\text { CMOS }\end{array}$ \\
\hline Diff. Gain (dB) & 60 & 32 & 30 & 50 & 38 & 42 \\
\hline $\mathrm{BW}(\mathrm{GHz})$ & $>15$ & 3 & 6.5 & 9.4 & 26.2 & 9.0 \\
\hline Sensitivity & $3.5 \mathrm{mV}_{\mathrm{pp}}$ & $2.2 \mathrm{mV}_{\mathrm{pp}}$ & $25 \mathrm{mV}_{\mathrm{pp}}$ & $4.6 \mathrm{mV}_{\mathrm{pp}}$ & $20 m V_{p p}$ & $10 m V_{p p}$ \\
\hline BER & $10^{-9}$ & $10^{-12}$ & - & $10^{-12}$ & $10^{-7}$ & $10^{-12}$ \\
\hline Inductors & No & No & Yes & Yes & Yes & No \\
\hline VDD (V) & $3.5-5.5$ & 2.5 & 2.4 & 1.8 & 1.5 & 1.8 \\
\hline Power (mW) & 600 & 53 & 120 & 150 & 125 & 189 \\
\hline Area $\left(\mathrm{mm}^{2}\right)$ & $1.2 \times 2.6$ & $0.2 \times 0.15$ & $0.6 \times 0.65$ & $0.5 \times 1.5$ & $0.3 \times 1.3$ & $0.32 \times 0.6$ \\
\hline
\end{tabular}

\section{REFERENCES}

[1] Y. M. Greshishchev and P. Schvan, "A 60-dB gain, 55-dB dynamic range, $10-\mathrm{Gb} / \mathrm{s}$ broadband SiGe HBT limiting amplifier," IEEE $J$. Solid-State Circuits, vol. 34, no. 12, pp. 1914-1920, Dec. 1999.

[2] C. Q. Wu, E. A. Sovero, and B. Massey, " $40 \mathrm{GHz}$ transimpedance amplifier with differential outputs using InP/InGaAs heterojunction bipolar transistors," in Symp. GaAs IC Dig. Tech. Papers, Oct. 2002, pp. 60-63.

[3] H. Tran, F. Pera, D. S. McPherson, D. Viorel, and S. P. Voinigescu, "6-k $\Omega$ 43-Gb/s differential transimpedance-limiting amplifier with auto-zero feedback and high dynamic range," IEEE J. Solid-State Circuits, vol. 39, no. 10, pp. 1680-1689, Oct. 2004.

[4] E. Säckinger and W. C. Fischer, "A 3-GHz 32-dB CMOS limiting amplifier for SONET OC-48 receiver," IEEE J. Solid-State Circuits, vol. 35 , no. 12 , pp. $1884-1888$, Dec. 2000.

[5] C. H. Wu, J. W. Liao, and S. I. Liu, "A 1 V $4.2 \mathrm{~mW}$ fully integrated 2.5 $\mathrm{Gb} / \mathrm{s}$ CMOS limiting amplifier using folded active inductors," in Proc. IEEE ISCAS, May 2004, vol. 1, pp. 1044-1047.

[6] C. H. Wu, C. S. Liu, and S. I. Liu, "A 2 GHz CMOS variable-gain amplifier with $50 \mathrm{~dB}$ linear-in-magnitude controlled gain range for 10 GBase-LX4 Ethernet," in IEEE ISSCC Dig. Tech. Papers, Feb. 2004, pp. $484-485$.

[7] H. C. Tsai, J. Y. Yeh, W. H. Tu, T. C. Lee, and C. K. Wang, "A 10 GBase-LX4 receiver front end transimpedance amplifier and limiting amplifier," in Proc. IEEE International Symposium on Circuits and Systems, May 2004, vol. 4, pp. 393-396.

[8] R. Tao and M. Berroth, " $10 \mathrm{~Gb} / \mathrm{s}$ CMOS limiting amplifier for optical links," in Proc. ESSCIRC, Sep. 2003, pp. 285-287.

[9] W. Z. Chen, Y. L. Cheng, and D. S. Lin, "A 1.8-V 10-Gb/s fully integrated CMOS optical receiver analog front-end," IEEE J. Solid-State Circuits, vol. 40, no. 6, pp. 1388-1396, Jun. 2005.

[10] S. Galal and B. Razavi, "10-Gb/s limiting amplifier and laser/modulator driver in $0.18 \mu \mathrm{m}$ CMOS technology," IEEE J. Solid-State Circuits, vol. 38, no. 12, pp. 2138-2146, Dec. 2003.

[11] C.-H. Lee and S.-I. Liu, "A 35-Gb/s limiting amplifier in $0.13-\mu \mathrm{m}$ CMOS technology," in Symp. VLSI Circuits Dig. Tech. Papers, Jun. 2006, pp. 152-153.

[12] R. P. Jindal, "Gigahertz-band high-gain low-noise AGC amplifiers in fine-line NMOS," IEEE J. Solid-State Circuits, vol. SC-22, pp. 512-521, Aug. 1987.

[13] B. Analui and A. Hajimiri, "Bandwidth enhancement for transimpedance amplifiers," IEEE J. Solid-State Circuits, vol. 39, no. 8, pp. 1263-1270, Aug. 2004.

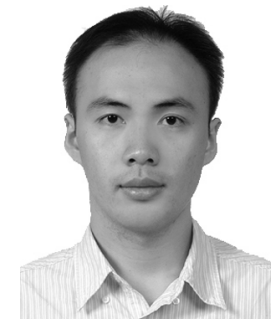

Huei-Yan Huang was born in Taipei, Taiwan, R.O.C., in 1982. He received the B.S. and M.S. degrees in electronics engineering from National Taiwan University, Taipei, in 2004 and 2006, respectively.

His research interests focus on integrated circuit designs for broadband communication systems.

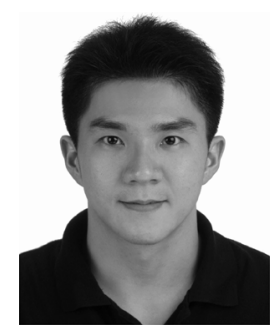

Jun-Chau Chien (S'05) was born in Taipei, Taiwan, R.O.C., in 1981. He received the B.S. and M.S. degrees in electronics engineering from National Taiwan University, Taipei, in 2004 and 2006, respectively.

His research interests focus on integrated circuit designs for high-speed communication systems.

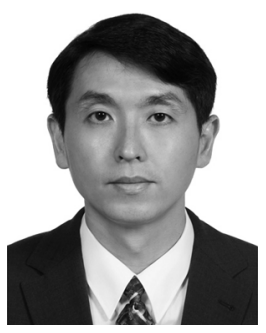

Liang-Hung Lu (M'02) was born in Taipei, Taiwan, R.O.C., in 1968. He received the B.S. and M.S. degrees in electronics engineering from National Chiao-Tung University, Hsinchu, Taiwan, in 1991 and 1993, respectively, and the Ph.D. degree in electrical engineering from the University of Michigan, Ann Arbor, in 2001. During his graduate study, he was involved in SiGe HBT technology and monolithic microwave integrated circuit (MMIC) designs.

From 2001 to 2002, he was with the IBM T. J. Watson Research Center, Yorktown Heights, NY, working on low-power and RF integrated circuits for silicon-on-insulator (SOI) technology. In the August 2002, he joined the faculty of the Graduate Institute of Electronics Engineering and the Department of Electrical Engineering, National Taiwan University, Taipei, Taiwan, where he is currently an Associate Professor. His research interests include CMOS/BiCMOS RF and mixed-signal integrated circuit designs. 